\title{
La astucia en la conceptualización platónica de бoфía ${ }^{1}$
}

\section{The Element of Cunning in Plato's Conceptualization of бoфía}

\author{
Trinidad SilVA IRARRÁZAVAL \\ https://orcid.org/0000-0002-1424-5039 \\ Universidad Alberto Hurtado, Chile \\ tsilvai@uahurtado.cl
}

RESUMEN: Considerando el lugar que tiene la astucia en la caracterización de бoфía en la tradición griega antigua, desde la literatura del período arcaico hasta el pensamiento del círculo socrático, llama la atención que en Platón no exista algo como el ooфós astuto. Aparte del Hipias Menor, que presenta una

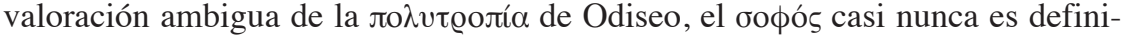
do por su inteligencia -ésta no es una característica distintiva del бoфó o del

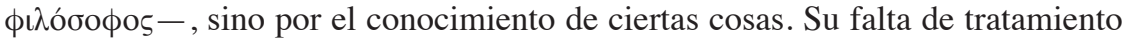
ha significado un abandono del tema de parte de la mayoría de los intérpretes. A fin de remediar esta situación, en este artículo ofrezco una interpretación que diagnostica la ausencia de un atributo como la astucia en la conceptualización de ooфía en Platón, pero no como el resultado de simple condena o censura como sostienen, por ejemplo, Detienne y Vernant 1978 y sugiere Montiglio 2011. En este trabajo propongo que Platón manifestaría una falta de interés con respecto al tratamiento de estos atributos. A partir del análisis del corpus platónico busco demostrar que los atributos de la inteligencia, si bien son considerados cualidades ventajosas y deseables, tienen nada más un valor instrumental con respecto a la consecución de la verdad y del bien.

Palabras clave: astucia, ooфía, Platón, Antístenes, Odiseo

AвSTRACT: Considering the importance of cunning in the characterization of бoфía in the Ancient Greek tradition, from the literature of the archaic period to the Socratic circle, it is striking that in Plato there is no such thing as a cunning

\footnotetext{
${ }^{1}$ Este tema se desarrolló como parte de la tesis doctoral en el Departamento de Griego y Latín del University College London (cf. Silva 2017) y se presentó como ponencia en la Conferencia SOCRATICA IV, Buenos Aires, 2018. El artículo se realizó en el marco del Proyecto Conicyt PAI Convocatoria Nacional Chile Subvención a Instalación en la Academia Convocatoria año 2018 PAI77180035.
} 
бoфós. Apart from the Lesser Hippias, which offers an ambiguous assessment

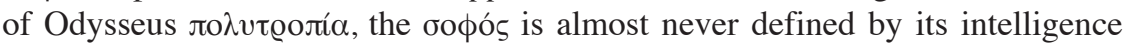

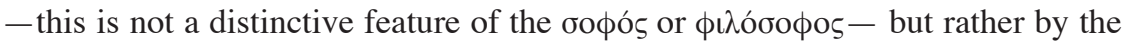
knowledge of certain things. The lack of treatment has led to most interpreters to neglect the subject. In order to remedy this situation, in this article I offer an interpretation that diagnoses the absence of an attribute such as cunning in the conceptualization of бoфí $\alpha$ in Plato, but not as the result of simple condemnation or censorship as argued, for example, by Detienne and Vernant 1978 and suggested by Montiglio 2011. In this paper I propose that Plato would manifest a lack of interest regarding these attributes. From the analysis of the Platonic corpus I seek to demonstrate that, although attributes of intelligence are considered advantageous and desirable qualities, they have only instrumental value with respect to the attainment of truth and good.

Keywords: Cunning, ooфía, Plato, Antisthenes, Odysseus

RECIBIDO: 20/03/2019 • AcEPTADO: 05/06/2019 • VERSIÓn FINAL: 07/08/2019

Las características del ingenio que designa ooфía se hallan en la poesía de Teognis, en las Historias de Heródoto y en los autores trágicos. Muchos de estos ejemplos son testimonio de que en la literatura pre-platónica ooфí $\alpha$ puede asociarse con astucia, ingenio, habilidad y destreza, tal como lo desig-

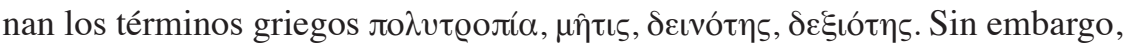
Platón no menciona dichos atributos en ninguna de sus caracterizaciones o descripciones más importantes de ooфós y бoфía. Es más, no parece incluirlas en su filosofía. Esto sorprende si se considera el lugar ejemplar que tiene

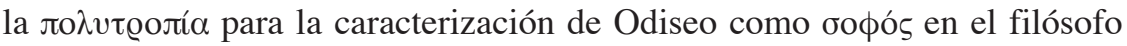
socrático Antístenes (Porph., ad Od., 1, 1, ed. 1890). El presente análisis ofrece una interpretación que diagnostica la falta de un atributo como la astucia en la conceptualización de ooфí $\alpha$ en Platón, no como el resultado de simple condena o censura - porque se aleja de la verdad filosófica o es inmoral-, como afirman, por ejemplo, Detienne y Vernant 1978 y deja entrever Montiglio 2011. Con respecto al tratamiento de estos atributos, nosotros proponemos que, más que censura o condena, Platón manifiesta un desinterés.

Para lograr nuestro objetivo, comenzaremos por establecer dos aspectos importantes sobre бoфía/бoфós en la literatura antigua: i) la terminología tiene un claro sentido honorífico, pero su significado es contencioso; y ii) la terminología designa la capacidad intelectual en un sentido amplio y neutro, de modo que, según el contexto, puede adquirir tonos evaluativos positivos o negativos. Esto es relevante para este análisis en la medida en que demuestra que tanto Platón como Antístenes, de distintas maneras, deben justificar el compromiso de la ooфía con la verdad y el bien. Atendiendo 
este compromiso, la presente investigación busca demostrar que Platón no conceptualiza ooфí $\alpha$ en términos de inteligencia o astucia, no porque atente contra la verdad o el bien, sino porque como mera capacidad o atributo del carácter no garantiza nada. La tesis principal se justifica al hacer una distinción entre conocimiento, entendido como saber acerca de algo, e inteligencia, atributo del carácter.

\section{EL VALOR FILOSÓFICO DE इOФIA}

Antes de determinar hasta qué punto ooфía se conceptualiza como astucia o inteligencia e indicar su valor filosófico, es necesario establecer algunos antecedentes que definen su relevancia en la literatura antigua. Lo que primero se ha de advertir sobre ooфía es la ausencia de un equivalente en castellano. Estimamos que la traducción estandarizada de 'sabiduría' se presta a equívocos, cuando el campo semántico incluye 'conocimiento', 'inteligencia', 'habilidad', 'buen juicio', 'experticia', 'astucia', 'agudeza', 'ingenio', 'sagacidad' y 'perspicacia', etcétera. Si bien éstas son cualidades de la mente, hay una diferencia fundamental entre ser astuto y sabio, entre ser experto en una materia y ser sagaz o tener buen juicio. Tal distinción será fundamental para entender la presente propuesta.

Sobre este punto, se ha de tener en cuenta que la posibilidad de reconstruir un significado único o transversal de ooфía queda limitado no sólo por las dificultades propias de la traducción, sino también por la naturaleza agonística de la terminología en juego. Como sea que estemos tentados a reconstruir el significado e importancia de ooфía o бoфós, debemos empezar por considerar que la noción es sobre todo usada en el contexto de la competencia. Esto ya lo advierte Lloyd en su sobresaliente libro The Revolutions of Wisdom: "Rivalry in claims to be wise starts almost as soon as we have any evidence to go on in Greece, and what counted as wisdom was an extraordinarily open-ended and negotiable question" (Lloyd 1987, p.

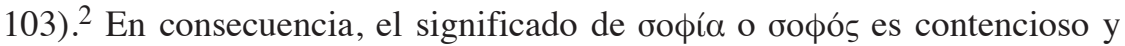
debe interpretarse atendiendo a cada contexto. ${ }^{3}$ Precisamente porque, como título honorífico, ooфós tiene un sentido adaptable y negociable, las condiciones del ảyóv no están reservadas a un género o área específica; pues las manifestaciones de rivalidad en ooфí $\alpha$ son intra e intergenéricas. ${ }^{4}$ Ejemplos

\footnotetext{
${ }^{2}$ Nuestra traducción: "La rivalidad entre declaraciones de sabiduría en Grecia empieza tan temprano como lo demuestra la evidencia, y lo que cuenta como sabiduría era algo extremadamente abierto y negociable".

${ }^{3}$ Para este tema en relación con Platón, cf. Nehamas 1990; Nightingale 1995; Tell 2011,p. 17.

${ }^{4}$ Aunque se puede entender de modo generalizado como una competencia por el título de бoфós. Cf. Nightingale 2000, p. 157.
} 
de este fenómeno aparecen en Teognis contra Academo (993-6, ed. 1931), Jenófanes contra los atletas (DK B11-14, ed. 1951), Heráclito contra Homero (DK B42, B56) y contra Hesíodo (DK B57) e Hipócrates contra Empédocles y otros pensadores (VM, 20,1-6, ed. 1990). Teniendo esto en cuenta, es quizá más relevante observar el sentido honorífico en vez de las distintas acepciones de la terminología en cuestión.

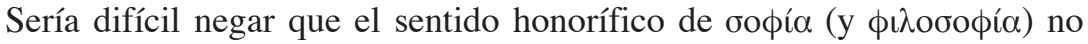
alcanza su punto más alto con la apropiación que hacen, de diversas maneras, Platón y Aristóteles en el s. IV a. C. En el caso de Platón, бoфía/бoфós está en el centro de la caracterización de Sócrates contra las primeras acusaciones (Ap., 17a-23c, ed. 1900), las discusiones morales sobre la virtud (La., 194d1 ss., ed. 1903; Prt., 352b1 ss., ed. 1903; Euthd., 278d-282e, ed. 1903, y $R$., 427e-443e, ed. 2003) y sobre el conocimiento (Tht., 145d-e, y 161d-179b, ed. 1900). Pero, a diferencia de Aristóteles, quien distingue a las virtudes intelectuales de las éticas, Platón considera que la ooфí $\alpha$ está vinculada tanto con la virtud moral como con la intelectual, es decir que

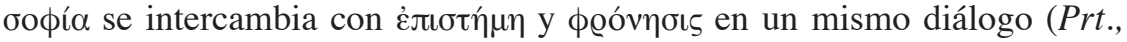
$352 \mathrm{c} 7$ y $352 \mathrm{~d} 1$; Euthd., $281 \mathrm{~b} 2$ y $281 \mathrm{~b} 6$; Tht., $145 \mathrm{e} 6$ y 176b3). Es la virtud propia de la clase guardiana en $R$., 429a1-3, y la totalidad de la virtud (donde equivale al conocimiento de lo bueno y malo) en $L a$., 199c7, y Prt., 360d4-5. Aristóteles, en cambio, hace una clara taxonomía de las virtudes y distingue a las éticas de las intelectuales (EN, 1139b16, ed. 1894: $\tau \dot{\varepsilon} \chi v \eta$,

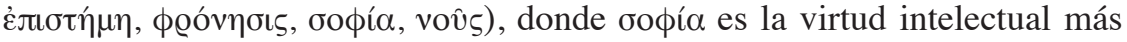

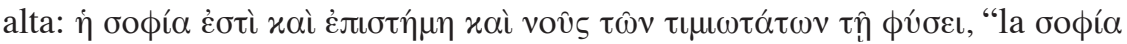
es ciencia e intelecto de las cosas más honorables por naturaleza" (Arist., $E N, 1141 \mathrm{~b} 2-3$, trad. nuestra). Esta concepción no sólo es consistente en el resto de la obra, particularmente en el libro X (EN, 1179a30), sino también en Metaph. (981b27, 982a4, 982a12 y 983a5, ed. 1924), y Peri Philosophias (fr. 8b Ross, ed. 1952).

En Platón no encontramos este tipo de definición sistemática establecida desde una distinción entre el plano práctico y teórico; más bien, hallamos una conceptualización de ooфía vinculada esencial e indistintamente con la verdad y el bien. No sobra decir que esta indistinción rescata la valoración ética del conocimiento que existe, en buena parte, como legado del intelectualismo socrático. La consecuencia inmediata es que el valor filosófico de бoфía en Platón está marcado por un compromiso con el conocimiento verdadero y con el comportamiento recto. Cabe destacar, para el propósito del presente estudio, que este valor filosófico se aplica en el caso de los dos filósofos socráticos: Antístenes y Platón. La diferencia radica en los fundamentos ontológicos y epistemológicos de cada sistema que, en el caso de Antístenes, permiten que бoфía se conceptualice en términos de astucia y versatilidad, y en el caso de Platón no. 


\section{El SENTIDO EVALUATIVO DE হOФIA}

Es necesario entender que el valor filosófico de ooфía, que supone un compromiso epistemológico con la verdad y un compromiso ético con el bien, no es un fenómeno generalizado en la literatura pre-platónica. Quizás el único precedente es Heráclito, quien en uno de los fragmentos declara:

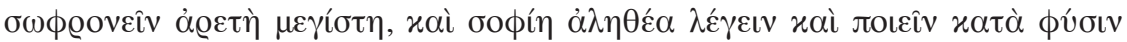

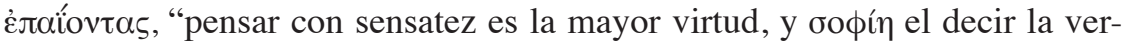
dad y actuar de acuerdo a la naturaleza, atendiéndola" (DK B112). Heráclito, entre los presocráticos, es sin duda el que más atendió esta noción, y puede haber sido un precedente importante para Platón en más de un sentido (DK B32). ${ }^{6}$ En la mayoría de las ocasiones, se observa que бoфía designa una competencia intelectual neutra cuya aplicación y propósito, según el caso, la pueden convertir en algo loable o condenable. इoфía y бoфós, comprendidos como términos que designan inteligencia, experticia o conocimiento, bien pueden utilizarse como epítetos de elogio o desaprobación. Hay varios ejemplos de esto.

Píndaro usa regularmente esta terminología para describir la habilidad del poeta ( $P ., 9,78$, y 10, 22, ed. 1997; I., 7, 18; 1, 45; y 8, 47, ed. 1997). Los hombres devienen ooфoí y à $\gamma \alpha \theta$ oí de acuerdo con la divinidad $(O ., 9$,

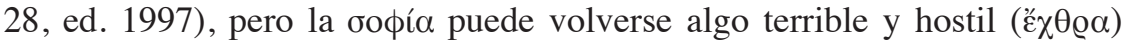
cuando cuestiona el estatus inmortal de la deidad, atribuyéndole acciones innobles $(O ., 9,38)$. En una de sus elegías, el poeta Teognis exhorta a Cirno para que actúe como un pulpo enredado $(1,214: \pi 0 \lambda u ́ \pi \lambda$ oxos), admitiendo

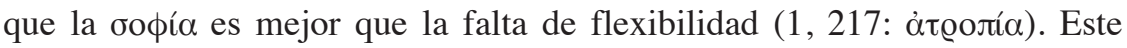
ideal de versatilidad e ingenio que Teognis aconseja se reformula en otro pasaje para declarar que la ooфía es incluso mejor que la gran virtud (1,

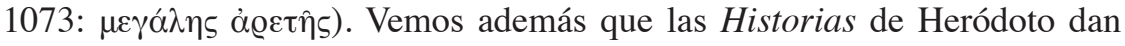
testimonio de que Solón se distingue por su ooфía en virtud de su experiencia y conocimiento (Hdt., 1, 30, 2, ed. 1932), pero también se reconoce el ingenio y talento detrás del robo del tesoro del rey Rampsinito; si bien es

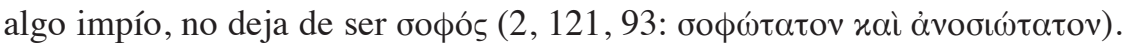
El contraste resulta más claro en las Bacantes de Eurípides con la fórmula

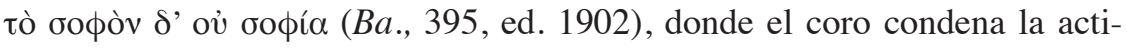
tud crítica de Penteo con respecto a la celebración de los ritos dionisiacos. La contraposición de los dos sentidos de ooфía es evidente sobre todo en cuanto el término vale como epíteto de aprobación para la sabiduría y el consejo de Tiresias $(B a ., 179,183)$ y de desaprobación para la actitud crítica de Penteo (Ba., 203). En general ooфós es un título de autoridad y buena

\footnotetext{
${ }^{5}$ Trad. de García Gual 2018, p. 34.

${ }^{6}$ Esta terminología tiene un claro estatus especial en Heráclito, cf. DK B83, B108 y B112.
} 
reputación, pero en las Nubes de Aristófanes los ooфoí representan lo peor de la clase intelectual, con un tipo de saber inútil, superficial y ajeno a la tradición ( $C l ., 94$, ed. 1968). Es así como la figura del astuto, el ingenioso o el intelectual, encarnado en el ooфós, si bien ocupa un lugar significativo en la literatura antigua desde Píndaro hasta Eurípides, no siempre goza de aprobación. ${ }^{7}$ Es importante recordar esto porque la astucia y, en general, la competencia intelectual que designa ooфí $\alpha$ no están naturalmente asociadas con la aprobación moral, sino que merecen justificarse. Dado este contexto, se entiende en mayor grado cuáles son las condiciones que determinan el desafío de rehabilitar la ooфí $\alpha$ de Odiseo en Antístenes, o la ooфía de Sócrates en Platón, de modo que su sentido honorífico de compromiso con la verdad y el bien quede asegurado.

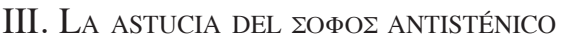

Aunque en la Odisea, el espíritu versátil del protagonista es evaluado como una clara cualidad (13, 291 ss., ed. 1962), en la poesía post-homérica del s. v a. C. este mismo espíritu es objeto de crítica, sobre todo en el retrato que ofrece Sófocles en Filoctetes. Teniendo esto en cuenta, vale la pena observar cómo surge al interior del círculo socrático un intento de rehabilitar tanto la figura del Laertíada como su ingenio. Si bien es discutible de qué manera Odiseo se presenta entre los seguidores de Sócrates, todo indica que este héroe homérico es un referente esencial para el maestro. En este caso, nos interesa destacar el ejemplo de Antístenes porque, como apunta la evidencia textual, es el único que reivindica abiertamente la astucia de Odiseo de su mala fama y la convierte en una sabiduría relevante para la filosofía. Decimos que es el único ya que si bien en la tradición filosófica, incluso en Platón, se rescata y valora la virtud del Laertíada desde su perseverancia, pobreza, autocontrol y fortaleza, como lo hace después Séneca ( $E p$., 88, ed. 1920), no se realiza abiertamente alabando su ingenio o astucia. ${ }^{8}$ An-

${ }^{7}$ El fenómeno de la hostilidad contra los intelectuales a finales del s. v a. C. obedece a distintos factores. Históricamente, puede explicarse por la convergencia de la guerra, la plaga, la alienación de la clase intelectual de la vida política y la inestabilidad que sufre la democracia en manos de la oligarquía (en los años 411 y 404 a. C.). Acerca de este tema, cf. Todd 1993 y Wallace 1994. El clima antintelectual también se explica a partir de un desplazamiento del paradigma cultural, sobre todo como efecto de la alfabetización. Ford 2002 la describe como el paso a un nuevo modelo de clase intelectual, donde la tradición oral es desplazada por el artefacto escrito, donde la figura del poeta es desplazada por la del pensador, donde lo nuevo desplaza a lo viejo. Dentro de este contexto, el intelectual, el бoфós, como Penteo, Sócrates, Medea y Odiseo, es blanco de crítica por subyugar valores tradicionales.

${ }^{8}$ La presentación de Odiseo en estos términos no debiera sorprendernos, ya que es fiel a la caracterización homérica en varios puntos. Además de las habilidades oratorias e intelectuales 
tístenes hace esto desde su doctrina. El principio antisténico que opera en estos pasajes es el de verdad onomástica: en la medida en que cada cosa

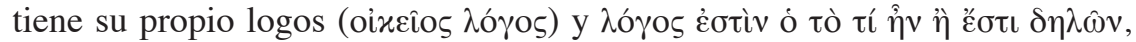
el logos se entiende como "lo que muestra lo que algo era o es" (D. L., VI, 3, 1-2= SSR V.A 151, ed. 1966), el conocimiento en el plano lingüístico asegura el conocimiento en el plano ontológico, de modo que, si se habla con sentido, se habla necesariamente con la verdad y el que dice algo, dice lo que es. ${ }^{9}$ Este principio, que justifica la importancia del lenguaje y del método de investigación de los nombres, permite que Odiseo no sea nada más un ejemplo de genio astuto, sino también de ooфós, pues en virtud de su

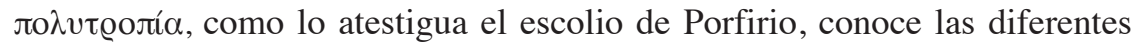
formas de discurso:

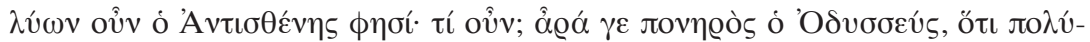

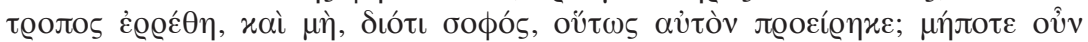

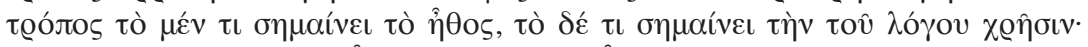

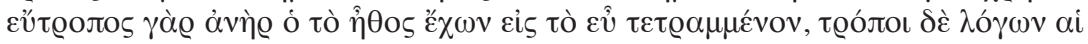

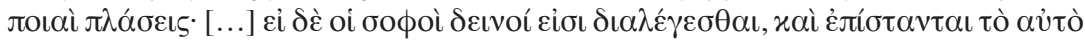

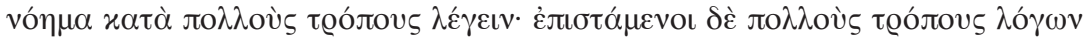

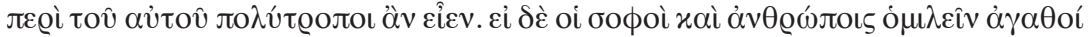

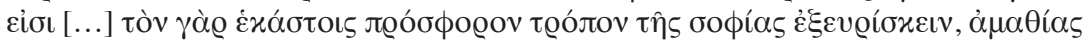

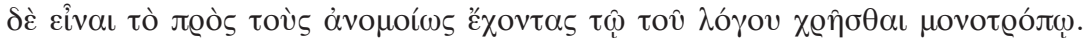

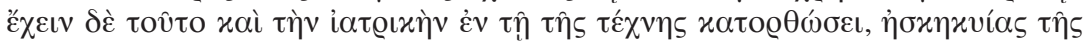

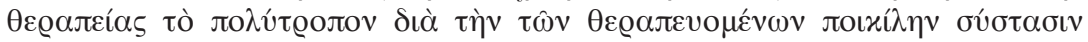
(Porph., ad Od., XXXIII, 337, ed. 1890).

Entonces, al analizar dice Antístenes: “¿y entonces qué? ¿Acaso es malvado Odiseo porque fue llamado polútropos, y no <es posible pensar que Homero> lo llamó así porque era sabio? ¿Acaso trópos no significa en un <verso $>$ el carácter y en otro significa el uso del discurso? Pues eútropos es el varón que tiene el carácter vuelto hacia el bien, y trópoi de los discursos son los varios estilos? [...] Y si los sabios son hábiles para hablar, saben decir el mismo concepto de muchos modos y conociendo muchos modos de argumentos sobre los mismos serían polútropoi. Los sabios son buenos para convivir con los hombres. [...] Es propio de la sabiduría encontrar el modo de sabiduría apropiado para cada uno, mientras que es ignorancia valerse de un único modo de discurso frente a los que son disímiles. Es así también la medicina en la cura, cuando ejercita la variedad de modos de la terapia a través de la recomendación variada de elementos terapeúticos (trad. de Mársico 2011).

que usa a su favor, este héroe es un modelo de racionalidad y resiliencia. Uno de sus epítetos es también ro $\lambda u ́ \tau \lambda \alpha \varsigma$, 'de alta resistencia', cf. Hom., Il., 8, 97, ed. 1998; Od., 5, 171, ed. 1962; S., Aj., 956, ed. 1958.

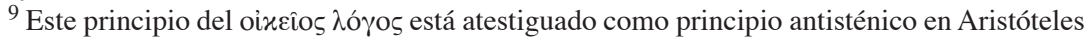
cuando evalúa la imposibilidad de la falsedad y la contradicción que resultan de ello (Metaph., 1024b32-34). Sobre el tema, cf. Brancacci 1990. 


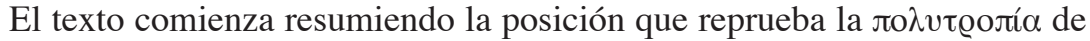
Odiseo que, en contraste con la simplicidad de Ayante, la sabiduría de Néstor y la honestidad de Aquiles, se asocia con un carácter "traicionero e inestable". Antístenes, dice Porfirio, desarticula esta concepción por medio de un argumento en el que pueden identificarse tres momentos: $i$ ) uno de disociación, ii) otro de re-asociación y finalmente iii) uno de aprobación.

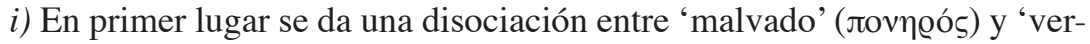

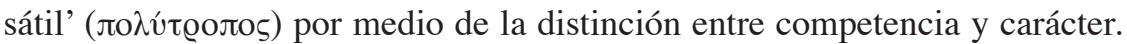

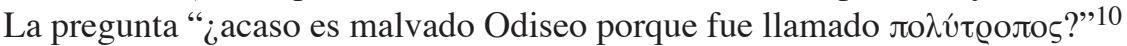
indica que la asociación asumida en un comienzo puede ser cuestionada. Esto lo hace Antístenes al analizar los distintos significados del componente

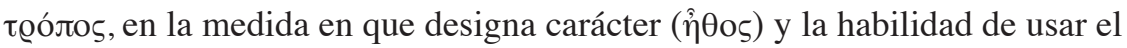

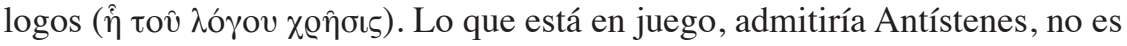
una evaluación de carácter, sino una habilidad que se traduce en un manejo del discurso y una capacidad para adaptarse a distintas situaciones.

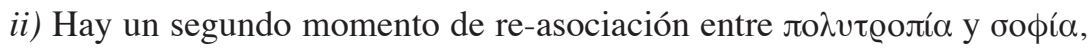
donde ooфía es claramente un epíteto de alabanza. Antístenes responde a la pregunta anterior del siguiente modo: "Es posible que Homero lo haya

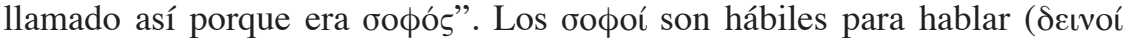

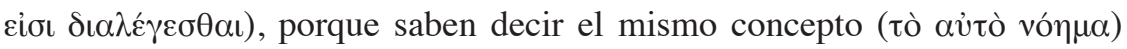
de muchas maneras y también saben relacionarse con la gente de muchas maneras. Las itálicas destacan la presencia del componente de multiplicidad y variedad ya que éste es precisamente el aspecto que Antístenes se propone

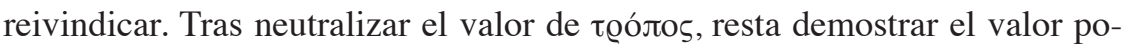
sitivo de lo múltiple y variado que designa roגús. Esto lo hace mediante la

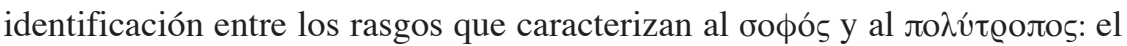
conocimiento de las distintas formas de discurso y la capacidad de adaptarse a los diferentes interlocutores y audiencias.

iii) Finalmente, hay un momento de aprobación. La versatilidad es un atributo que está al servicio del conocimiento y la verdad: "es propio de

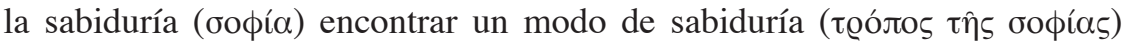
apropiado para cada uno, mientras que es propio de la ignorancia ( $\dot{\alpha} \mu \alpha \theta \dot{\alpha} \alpha)$

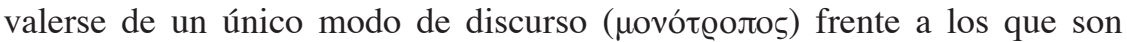
disímiles". La trascendencia filosófica de dicha habilidad está dada por la comparación con la medicina; el conocimiento del logos propio para cada cosa que concentra la variedad de lo existente permite que el discurso sea útil y beneficioso para la audiencia. La desaprobación se la lleva entonces

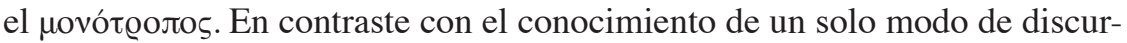
so, "la habilidad lingüística del hombre лодút@oлоऽ lo convierte en un sabio, ya que con su multiplicidad puede entender la trama variada de lo existente,

${ }^{10}$ Trad. de Mársico 2011. 
asignar a cada cosa el nombre que le es propio y, a la vez, adaptar el discurso al tipo de interlocutor que le toque en suerte" (Mársico 2011, p. 44).

Es importante observar, con respecto al último punto, que el conocimiento de las formas de discurso no se reduce a una habilidad para hablar. Como

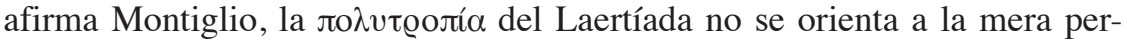
suasión de la audiencia, sino que impulsa al interlocutor a descubrir lo que es verdadero y beneficioso para él:

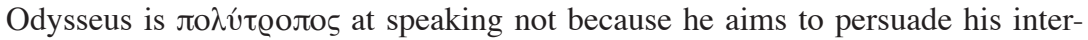
locutors of anything, in Gorgias' style, but because, thanks to his knowledge of a variety of modes of speech, he can push them to learn what is true and beneficial to them. The reference to medicine spells out that the бoфó $\varsigma$ speaker, far from resembling a Sophist, is concerned with therapy, and if he uses different techniques of persuasion, it is for the good of each individual patient (Montiglio 2011, p. 24). ${ }^{11}$

Vemos entonces que, en cuanto тœóлоร, puede interpretarse como una habilidad moralmente neutra, no hay modo de justificar la desaprobación de

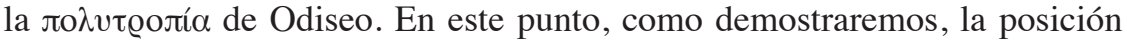
coincidiría con la que se defiende en el Hipias Menor de Platón. Pero Antístenes va más lejos aún, pues, en la medida en que el conocimiento de las diferentes formas de discurso implica un conocimiento de la realidad que aproxima a la verdad y al bien, esta habilidad sí merece aprobación moral.

\section{El zOфoz es el Que sabe: Platón}

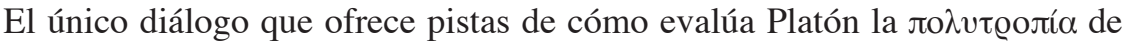
Odiseo está en el Hipias Menor. En una discusión sobre si el Laertíada o Aquiles es mejor, donde 'mejor' es definido en términos de habilidad, el diá-

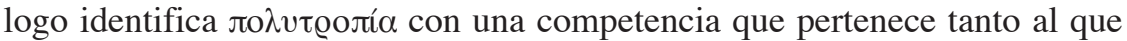
miente como al que dice la verdad. De esta manera, son cuestionados los supuestos que inicialmente asume Hipias, aquellos que vinculan la versatilidad con un carácter falso..$^{12}$ En este sentido, Platón realiza una disociación entre carácter moral y habilidad o competencia tal como Antístenes; pero, a diferencia de éste, no hace una re-asociación con ooфía identificada ésta

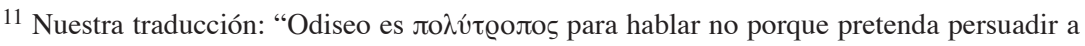
sus interlocutores de algo, al estilo de Gorgias, sino porque, gracias al conocimiento de la variedad de los modos de discurso, puede incitarlos a aprender lo que es verdadero y beneficioso para ellos. La referencia a la medicina explica que el hablante que es ooфós, lejos de parecerse a un sofista, está interesado en el efecto terapéutico y, si usa diferentes técnicas de persuasión, es por el bien de cada uno de los pacientes".

12 Para una revisión más completa y detallada del contenido filosófico de los diálogos, cf. Weiss 1981, Irwin 1988, Blundell 1992, Blondell 2002 y Adams 2010.
} 
como un atributo que resultaría exclusivamente asociado a un discurso verdadero o beneficioso. Así, ooфía se mantiene neutra como capacidad, en la medida en que puede aplicarse tanto para mentir como para decir la verdad. En el curso del diálogo, бoфía no se distingue de otras categorías intelectua-

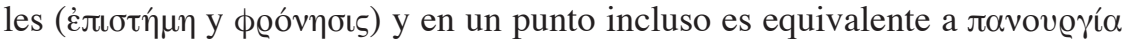
(Hp. Mi., 368e4, ed. 1903). Los matices evaluativos del término nada más son sugeridos al comienzo del Hipias Menor gracias a la caracterización de

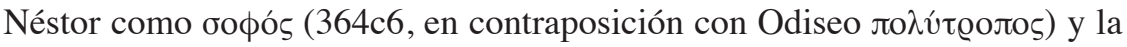
reputación de Hipias como un monumento de ooфía (364b2) en contraste con la confesión de ignorancia por parte de Sócrates (369d1 y 372b6). ${ }^{13}$ Mientras tenemos una idea de las connotaciones de ooфía a un nivel de caracterización dramática, el análisis de los argumentos centrales parece com-

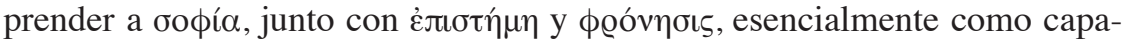

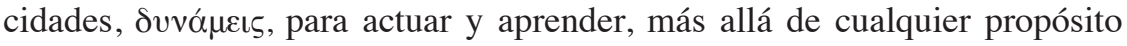
moral y compromiso con la verdad, todo eso lleva a consecuencias extremas que son indeseables tanto para Hipias como para Sócrates. ${ }^{14}$ Esto es así hasta el grado de admitir, hacia el final del diálogo, que quienes cometen injusticia voluntariamente y con conocimiento son mejores que quienes lo hacen involuntariamente y sin conocimiento (375d-376a).

En términos de caracterización dramática, en los diálogos de Platón, tanto Sócrates como los sofistas encarnan a los ooфoí del tipo ingenioso, al modo de Odiseo. ${ }^{15}$ A primera vista (y al menos desde la óptica de la opinión pública), ambos representan al tipo intelectual, el astuto que se sale con la suya, siempre sagaz, agudo, con múltiples recursos, artimañas y discursos. ${ }^{16}$ Pero esto sólo sucede a primera vista. Platón se encarga de establecer que en realidad ni su maestro ni los sofistas son verdaderos ooфoí: Sócrates es

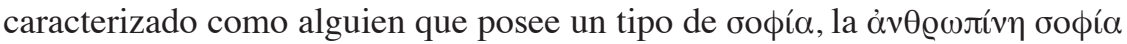
(Ap., 20d8), o bien como un фı $\lambda$ óøoфos; los sofistas son representados como

${ }^{13}$ Blondell 2002, p. 134, profundiza en la caracterización de Néstor como ooфós.

${ }^{14}$ Como plantea Adams 2010, p. 47, Sócrates no se propone evaluar el contenido moral (i.e. el valor) del objeto conocido ya sea por el hombre mentiroso o por el honesto, sino los medios a través de los cuales alcanzan sus propósitos.

${ }^{15}$ El término se aplica en la obra de Platón a reconocidos sofistas, oradores, sabios tradicionales y filósofos de la naturaleza. No hay que olvidar que ooфó ` y бoфía son títulos honoríficos atribuidos en gran medida por reputación que se origina en la opinión pública. Parte de la actividad filosófica de Sócrates en Platón, claramente enunciada en la Apología, es precisamente ir hacia aquellos que son reputados o creen ser expertos. Por eso, muchas menciones de los reputados ooфoí en Platón tienen que interpretarse a la luz de la ironía socrática. Algunos ejemplos son: Hipias (Hp. Mi., 364b1, ed. 1903; Prt., 314c1, 337c6), Protágoras (Tht., 152b1, 160d9, 161d9, ed. 1900; Prt., 309d2, 341a9), Pródico (Tht., 151b5; Phdr., 267b6, ed. 1901; Prt., 314c1), Eutidemo y Dionisodoro (Euthd., 271c6), poetas (Phdr., 235c4; R., 335e9, ed. 1902), oradores (Phdr., 269b3), sabios (Ti., 20d8, ed. 1902) y filósofos de la naturaleza (Grg., 507e6, ed. 1903; Lg., 886d7; 888e8, ed. 1907).

${ }^{16}$ Con respecto a la caracterización de Sócrates como tal, cf. Kofman 1988. 
dueños de un saber que es nada más aparente. ${ }^{17}$ De hecho, cuando Sócrates

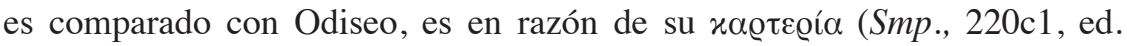

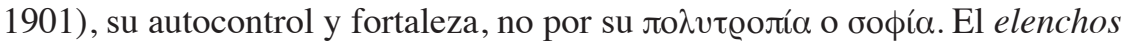
socrático en Platón muestra a un Sócrates que, si bien es recursivo y astuto, parte por reconocer su propia ignorancia y admite la posibilidad de descubrir la verdad en un esfuerzo conjunto con su interlocutor. ${ }^{18}$

Además del Hipias Menor, que presenta una evaluación ambigua de la

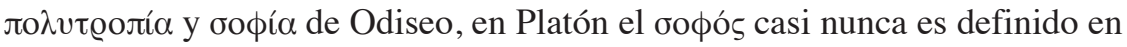
virtud de su astucia; ésta no es un atributo distintivo del verdadero ooфó $\mathrm{o}$ del filósofo. Algunos críticos interpretan la exclusión de tales características como un acto de censura o condena; pero sería injusto interpretar la falta de tratamiento como un acto de censura o condena. Detienne y Vernant, en su libro sobre $\mu$ ๆิtıs, afirman que Platón rechaza esta área de la inteligencia, lo que ellos llaman inteligencia estocástica, en nombre de la verdad filosófica:

Sophia becomes contemplative wisdom and ceases to refer to the knowledge of the skilful craftsman as it had ever since Homer's writings where Sophia was used of any organised body of knowledge with its own rules and methods handed on from one generation to another within professional groups such as those of the blacksmiths and carpenters [...]. The various forms of practical intelligence are sweepingly condemned once and for all in the name of the one and only Truth proclaimed by philosophy. The fact is that the Philosopher who appears as a sovereign arbiter of this classification is also responsible for the ephemeral definition which, so to speak, unifies the diverse forms of metis, bringing them together into a composite image presented in stark contrast to the immutable Knowledge claimed by a system of metaphysics based on Being and by the logic of Identity (Detienne \& Vernant 1978, pp. 315-316). ${ }^{19}$

No me parece que esta evaluación sea del todo acertada. En primer lugar, porque el dominio de la técnica, en particular la de los artesanos, demuestra

${ }^{17}$ La indistinción entre el sofista y el filósofo es un tema que claramente preocupa a Platón, como lo deja claro en Sph., 216c4-d2, ed. 1900. Los pasajes que ofrecen concepciones rivales de бoфí $\alpha$ entre Sócrates y los sofistas, principalmente Protágoras, se encuentran en Prt., 352b352d3, y Tht., 166d4-169d8.

${ }^{18}$ Cf. Montiglio 2011, p. 23.

${ }^{19}$ Nuestra traducción: "Sophia se transforma en sabiduría contemplativa y deja de referirse al conocimiento del hábil artesano como se había hecho desde los escritos de Homero, donde Sophia se usaba para designar un cuerpo organizado de conocimiento con sus propias reglas y métodos pasados de una generación a otra dentro de grupos profesionales, como el de los herreros y carpinteros [...]. Las varias formas de inteligencia práctica son condenadas de una vez por todas en el nombre de la única Verdad proclamada por la filosofía. El hecho es que el filósofo que aparece como el árbitro soberano de esta clasificación también es responsable de la definición efímera que, por así decir, unifica las distintas formas de metis, colocándolas juntas en una imagen compuesta que se presenta en crudo contraste con el Conocimiento inmutable proclamado por un sistema metafísico basado en el Ser y la lógica de la Identidad". 
ser reconocida, entre el arte de la política y el de la poesía, como ooфía en una obra tan importante como Ap., 22d3-4. ${ }^{20}$ En Laques, Protágoras, Eutidemo, República y Teeteto, Platón se sirve del modelo de distintas áreas de experticia, ejemplificado por carpinteros, flautistas, médicos y pilotos, a fin de demostrar que ooфía goza de cierto estándar de preparación y experiencia, fundamental para establecer un criterio de verdad no relativo. Por otro lado, si bien es verdad que Platón no incorpora el aspecto de la inteligencia estocástica, que incluye la astucia, la rapidez, la agudeza, tampoco lo condena. ${ }^{21}$ De hecho, en la República, cuando se define la naturaleza del filósofo, repite con frecuencia que el candidato ideal posee una serie de cualidades mentales, como la rapidez, la agudeza y la buena memoria. En el libro V, el filósofo es descrito a menudo como alguien con facilidad de aprendizaje

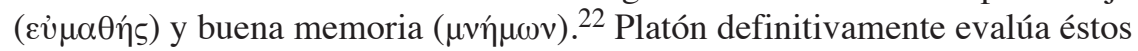
como atributos deseables en el filósofo, aún cuando reconoce que en general no son compatibles con los buenos temperamentos:

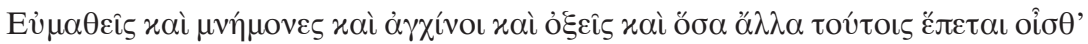

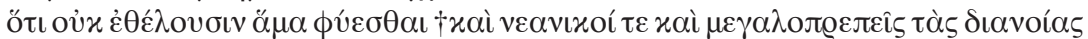

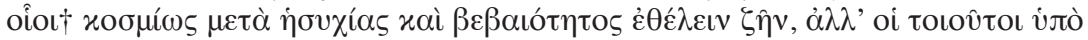

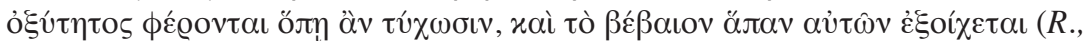
503c1-6, ed. 2003).

La facilidad de aprender, la memoria, la sagacidad, la vivacidad y cuantas cosas siguen a estas, el vigor mental y la grandeza de espíritu, no suelen crecer, bien lo sabes, junto con una disposición a vivir de una manera ordenada, con calma y constancia; sino que quienes las poseen son llevados azarosamente por su vivacidad y se les escapa todo lo constante..$^{23}$

Este tipo de evaluación se repite en el Teeteto, cuando Teodoro introduce a Teeteto como un estudiante excepcional en virtud de su rapidez para aprender y su buen carácter, dos atributos que en general no van juntos (Tht., 144a3). Este punto nos sirve sobre todo para comprender que no es que Platón rechace sin más los atributos de la inteligencia, sino que más bien los subordina o instrumentaliza. Montiglio capta la ambigüedad que hay en torno a estas cualidades en la obra de Platón:

\footnotetext{
${ }^{20}$ A diferencia de los políticos y los poetas, Sócrates reconoce que los artesanos son ooфú-

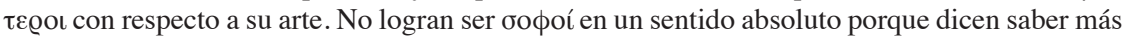
de lo que efectivamente saben.

${ }^{21}$ Por lo demás, identificar la inteligencia característica de Odiseo como estocástica también es discutible. Barnouw 2004, por ejemplo, defiende una conceptualización de la $\mu \eta \hat{\tau ı \varsigma ~ d e l ~ L a e r-~}$ tíada en términos de pensamiento reflexivo, capaz de anticipar y planificar.

${ }^{22}$ Cf. $R$., 486c3, 486d2, 487a3, 494b1-2, 503c1. Hay pasajes que consideran el movimiento y la rapidez como un aspecto positivo de las competencias intelectuales. Cf. Cra., 411d4, ed. 1900; Tht., 153b9, y Chrm., 160a1, ed. 1903.

${ }^{23}$ Trad. de Eggers Lan 1986.
} 
Plato's reluctance to endorse $\mu \hat{\eta} \tau \iota \varsigma$ suggests that he is caught between approval of that quality for its usefulness in the difficult navigation of life and philosophy and rejection of it on moral grounds. The hesitant promotion of Odysseus' ло $\lambda v \tau \varrho о \pi i \alpha$ in the Phaedrus and the Lesser Hippias, joint with the indictment of his actions and the questioning of his wisdom in the Apology, seems to indicate that Plato grappled with the commonplace view that condemned Odysseus' intelligence, eloquence, and inventiveness for their immoral applications, but ultimately did not settle on a positive evaluation of Odysseus against that view (Montiglio 2011, p. 47). ${ }^{24}$

No podemos sino estar de acuerdo con lo que asevera Montiglio, en particular con respecto a la inteligencia de Odiseo. La falta de tratamiento sistemático de estas cualidades en Platón es indiscutible. Sin embargo, no consideramos que la falta de tratamiento descanse en la censura moral, sino más bien en una cierta indiferencia. Nos atrevemos a sugerir que la razón por la que los atributos de inteligencia no tienen un lugar prominente en la filosofía de Platón es simplemente porque no juegan un rol significativo en ninguna de sus doctrinas. Ser inteligente, agudo y rápido son todos atributos deseables, pero podemos estar seguros de que Platón no pone a ooфía entre éstos. Incluso cuando no es central en su filosofía, la evidencia parece demostrar que Platón considera que los atributos propios de este tipo de inteligencia son: $i$ ) cualidades, $i i)$ no integrales al carácter moral de una persona, iii) en conflicto con otras cualidades. Resulta claro que, en el contexto de $R$., VI, dichas características son evaluadas positivamente por su potencial para facilitar el aprendizaje, mas vemos cómo en el libro VII - luego de que el símil de la caverna ha sido descrito y explicado, y la importancia de la

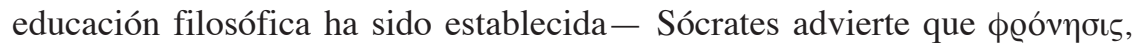

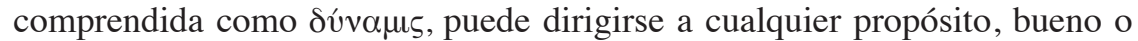
malo. Para ilustrar el caso, Sócrates ofrece el ejemplo de aquellos a los que

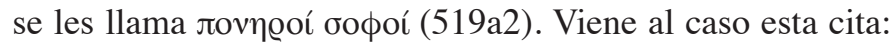

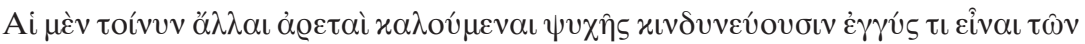

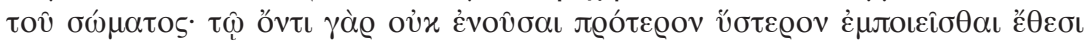

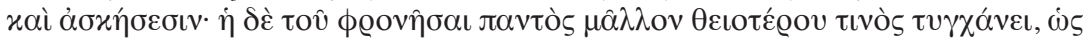

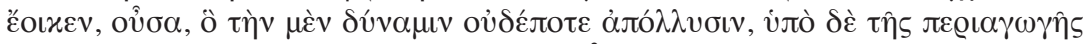

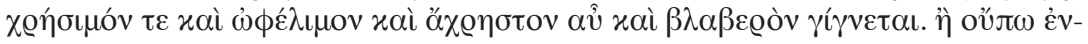

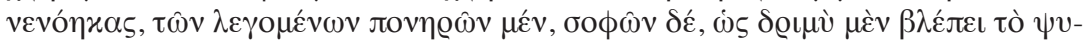

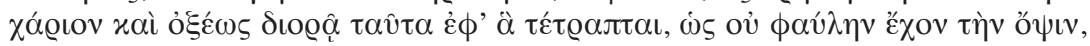

\footnotetext{
${ }^{24}$ Nuestra traducción: "La reticencia de Platón para incluir $\mu$ †ı sugiere que está atrapado entre la aprobación de esta cualidad por su uso en la difícil navegación de la vida y la filosofía,

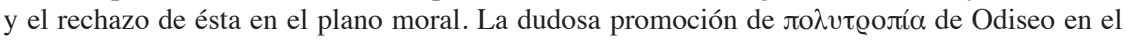
Fedro y el Hipias Menor, junto con la imputación de sus acciones y el cuestionamiento de su sabiduría en la Apología, parece indicar que Platón luchó contra el lugar común que condena la inteligencia de Odiseo por sus aplicaciones morales, pero finalmente tampoco se decidió a dar una evaluación positiva de Odiseo para cuestionar esa visión".
} 


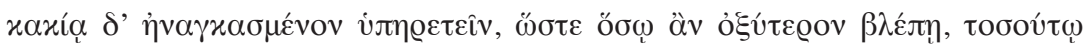

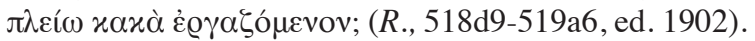

Ciertamente, las otras denominadas "excelencias" del alma parecen estar cerca de las del cuerpo, ya que, si no se hallan presentes previamente, pueden después ser implantadas por el hábito y el ejercicio; pero la excelencia del comprender da la impresión de corresponder más bien a algo más divino, que nunca pierde su poder, y que según hacia dónde sea dirigida es útil y provechosa, o bien inútil y perjudicial. ¿O acaso no te has percatado de que esos que son considerados malvados, aunque en realidad son astutos, poseen un alma que mira penetrantemente y ve con agudeza aquellas cosas a las que se dirige, porque no tiene la vista débil sino que está forzada a servir al mal, de modo que, cuanto más agudamente mira, tanto más mal produce ${ }^{25}$

La consecuencia inmediata es que la potencia de la inteligencia, incluso cuando se considera un instrumento fundamental para el aprendizaje, por sí misma no garantiza un uso adecuado, lo que en este contexto ilustra la importancia de la educación filosófica. El verdadero filósofo tiende hacia un objeto, lo uno, "lo en sî", la Idea.

\section{V. ГOФIA COMO CONOCIMIENTO}

Proponemos que la distinción relevante para comprender en mayor medida la apropiación platónica de ooфía es entre el ámbito del saber, entendido en un sentido amplio como el conocimiento de algo (teórico o práctico), y el ámbito de la inteligencia, entendido en un sentido amplio como capacidad mental. Por supuesto, estos ámbitos no son completamente separables, y es un tema controversial en la filosofía de la mente y en la teoría de la inteligencia. Sin embargo, sugerimos un criterio que está en la base de la propuesta de Gilbert Ryle en The Concept of Mind, uno de los primeros en abarcar este tema: cuando nos referimos a cualidades de la mente y llamamos a alguien 'agudo', 'rápido', 'perspicaz', 'astuto' o 'inventivo', la descripción no le atribuye el conocimiento de cierta verdad, sino la habilidad para hacer ciertas cosas: "It is of first-rate importance to notice from the start that stupidity is not the same thing, or the same sort of thing, as ignorance. There is no incompatibility between being well-informed and being silly, and a person who has a good nose for arguments and jokes may have a bad head for facts" (Ryle 1949, p. 26). ${ }^{26}$ Por lo mismo, dice Ryle: "[w]hen

\footnotetext{
${ }^{25}$ Trad. de Eggers Lan 1986.

${ }^{26}$ Nuestra traducción: "Es de primera importancia notar desde el comienzo que la estupidez no es la misma cosa, o el mismo tipo de cosa, que la ignorancia. No hay ninguna incompatibilidad entre estar bien informado y ser tonto, y una persona que tiene buen olfato para los argumentos y los chistes puede tener mala cabeza para los datos".
} 
a person is described by one or other of the intelligence-epithets such as 'shrewd' or 'silly', 'prudent' or 'imprudent', the description imputes to him not the knowledge, or ignorance, of this or that truth but the ability, or inability, to do certain sort of things" (Ryle 1949, p. 28). ${ }^{27}$ Esto significa que las manifestaciones de inteligencia no suponen un compromiso epistémico con la verdad. Ryle insiste en la diferencia porque cree que en la tradición filosófica se ha tramado lo que llama "leyenda intelectualista", la cual reduce las operaciones de la inteligencia a operaciones racionales de la mente. De esto se desprende una serie de distinciones y consecuencias que no es necesario tratar en este artículo. ${ }^{28}$

No sería ni justo ni acertado aplicar la distinción tal como la describe Ryle; de hecho, algunos dogmas de la leyenda intelectualista que dicho estudioso quiere desmitificar son imputables a Platón. ${ }^{29}$ Lo que propongo es que Platón advierte la diferencia básica entre cualidades de la mente y posesión de conocimiento, y privilegia una concepción de ooфí $\alpha$ en términos de lo segundo. Esto no significa que el aspecto de la inteligencia no esté presente. Tampoco implica que ooфía se reduzca a la comprensión de proposiciones verdaderas. La clave está en entender que, mientras el conocimiento tiene un correlato o un objeto determinado, la inteligencia se manifiesta en la habilidad o capacidad para hacer ciertas cosas. Si bien ambas pueden convergir - ciertamente la inteligencia se usa para la obtención, asimilación y aplicación de conocimiento - no se puede decir que la inteligencia se dirige necesariamente al conocimiento de cierta verdad. Esto es lo que importa para el análisis: бoфó $\varsigma$ y бoфía, como lo demuestran algunos ejemplos de la literatura antigua, pueden referirse a la astucia de alguien que logra engañar a otro, o a la rapidez de alguien que descifra un acertijo o el genio inventivo de quien planea una venganza; así, la posesión de cualidades mentales — ser rápido, agudo o astuto - no garantiza en ninguna medida el propósito para el que estas cualidades se aplican: para engañar o para ser injusto o deshonesto, o para cometer un crimen. Como afirma Kenneth James Dover en su libro sobre la moral popular en tiempos de Platón y Aristóteles:

Intelligence is indispensable for choosing the right means to an end, but the intelligent do not always choose good ends; a person of extraordinary ability in

\footnotetext{
${ }^{27}$ Nuestra traducción: "Cuando una persona es descrita por uno u otro epíteto de inteligencia como 'astuto' o 'tonto' o 'prudente' o 'imprudente', la descripción no le imputa conocimiento o ignorancia sino la capacidad o incapacidad para hacer ciertas cosas".

${ }^{28}$ Por ejemplo, el hecho de que la distinción entre inteligencia y conocimiento se establezca en términos de know-how y know-that.

${ }^{29}$ Entendida como conocimiento, бoфía incluye aspectos de know-how y know-that; en consecuencia, la distinción de Ryle 1945 y 1949 no es operativa en Platón. Dicha teoría hoy es debatida, en particular la demarcación entre know-how concebida como habilidad y know-that como conocimiento proposicional. Para profundizar en este tema, cf. Stanley 2011, Snowdon 2011 y Burnyeat 2011.
} 
the drawing of inferences, with an extraordinary sharp eye for relevance, may be selfish, cowardly, mean, callous or unreliable, while a halfwit may be helpful and kind and do his utmost to be good, with success so long as the complexities of a situation do not disguise the issue (1974, pp. 116-117) ${ }^{30}$

Si bien el conocimiento por sí mismo, esto es, saber más, tampoco compromete un único propósito en el contexto de la filosofía de Platón, la contemplación de ciertos objetos, por ejemplo, la Idea de Bien o de Belleza, no admite un ejercicio racional que pueda conducir al error o al vicio pues supone un compromiso epistemológico con la verdad y moral con el bien. $\mathrm{El}$ conocimiento es siempre conocimiento de algo, es siempre relacional. ${ }^{31} \mathrm{El}$ punto central del argumento es que al definir ooфía en términos de conocimiento, Platón puede preguntar "¿qué es lo que sabe el ooфóৎ?"32 La pregunta por el objeto de conocimiento (y su valor) es significativa porque permite a Platón calificar la ooфí $\alpha$, esto es crucial para distinguir la ooфí $\alpha$ de los diferentes expertos en sus distintos ámbitos: la ooфí $\alpha$ socrática, la ooфí $\alpha$ aparente y la бoфí $\alpha$ divina. Esto se deja ver especialmente en la Apología,

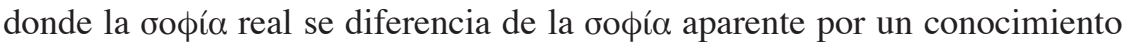
de las cosas más importantes o más valiosas (Ap., 21d4, 22c3, 22d7). Por nuestra parte, sugerimos que Platón mantiene el significado de ooфí $\alpha$ en la esfera del conocimiento, donde puede calificar el objeto de saber. Existen algunos patrones relevantes en los textos que dan cuenta de esto, a continuación enumeramos los más significativos:

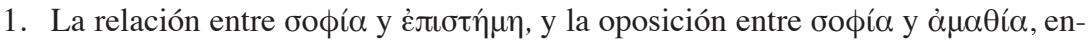
tendida como ignorancia o falta de conocimiento. ${ }^{33}$

${ }^{30}$ Nuestra traducción: "La inteligencia es indispensable para alcanzar los medios correctos para un propósito, pero la persona inteligente no siempre elige buenos propósitos; una persona con una habilidad extraordinaria para hacer inferencias, con un ojo extraordinariamente agudo para detectar relevancia, puede ser egoísta, cobarde, insensible, cruel o poco fiable, mientras una persona tonta puede ser servicial, amable y hacer todo lo posible para ser buena, con éxito en la medida en que las complejidades de la situación no oculten el problema".

${ }^{31}$ La $\delta o ́ \xi \alpha$, por supuesto, también es relacional. Éste no es un criterio que distingue al conocimiento de la $\delta$ ó $\xi \alpha$, pero sí al conocimiento de la inteligencia como atributo de carácter. Para que haya propiamente conocimiento tiene que existir al menos opinión verdadera, esto responde a ciertas condiciones que se discuten tanto en el Menón como en el Teeteto.

${ }^{32}$ Esto no desplaza la pregunta de cómo conoce el ooфó, i. e. las condiciones epistemológicas de verdad, que incluye los estados de creencia del sujeto. Pero sí es destacable la posición prioritaria del objeto del saber que garantiza en buena medida una aprehensión correcta y estable de la realidad. De esta manera, si bien puede admitirse la experticia de los artesanos como бoфía, ésta no constituye бoфía en el más alto grado, pues en Platón hay una valuación del objeto, de "las cosas más importantes" (cf. Ap., 22d4-e1).

33 Ésta no establece que haya sinonimia, si bien coinciden con cierta regularidad y en pasajes de importancia filosófica. Cf. e. g. Tht., 145d7-e7; R., 350c10-11, 429a1-3; Euthd., 275d4; Smp., 202a. 


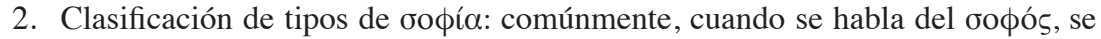
dice que es бoфós con respecto a algo. Así, el saber del бoфós se restringe a un área de conocimiento. Esto es importante para determinar, por ejemplo, que Sócrates es un tipo de ooфós, porque posee cierto conocimiento, o, en un caso similar, para distinguir al бoфós en sentido relativo, i. e. que demuestre un saber con respecto a algo (medicina, matemáticas, música, etcétera) del бoфós en sentido absoluto. ${ }^{34}$

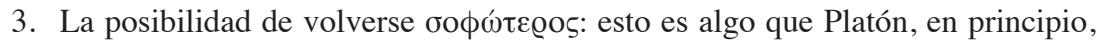
defiende, sobre todo en la medida en que garantiza la posibilidad de aprendizaje y también para ilustrar la importancia del estado intermedio del filósofo entre

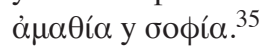

4. La caracterización del бoфó $\varsigma$ como intelectual: por lo general, en términos de caracterización, el ooфós da lugar a dos figuras, la del astuto, que se ve en Odiseo en la tradición post-homérica, y el ooфós intelectual, que encarna la figura de Sócrates en las Nubes de Aristófanes. Es importante distinguir esto, pues Platón se esfuerza más por reivindicar la figura del intelectual que personifica el ooфós. ${ }^{36}$

Nuestro argumento es que ninguna de estas características podría darse en una conceptualización de ooфí $\alpha$ como atributo de inteligencia.

\section{ConCLuSIÓN}

En el presente análisis hemos intentado dar una posible respuesta a la pregunta sobre el rol que tendría la astucia en la conceptualización platónica de ooфía, teniendo en cuenta que éste es un elemento que se encuentra claramente presente en representaciones de ooфí $\alpha$ y caracterizaciones del бoфós en la literatura griega, de modo conspicuo en el caso de Antístenes, filósofo socrático y rival teórico de Platón. El estudio comenzó por establecer algunos aspectos importantes en torno al significado de ooфí $\alpha$ y su valor filosófico. El punto relevante en esa sección fue determinar que ésta es una noción sumamente contenciosa, pero con un fuerte sentido honorífico, lo cual, en el contexto de la filosofía, obedece sobre todo a su compromiso con la verdad y el bien.

Luego se observó que la astucia, la versatilidad, i. e. la inteligencia como atributo de carácter que representa Odiseo de manera icónica en la tradición,

\footnotetext{
${ }^{34}$ Cf. Pl., Tht., $145 \mathrm{e} 3$.

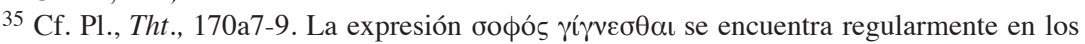
diálogos. Cf. Phd., 90c2; Tht., 145d8, 173b3; Stat., 290b2, ed. 1900; Phlb., 17e1, ed. 1901; Smp., 204a1; Ly., 210d1, ed. 1903; Euthd., 282b6, 282e5, 283b5, 283c6, 283d1; Alc., 118c4, 119a3, ed. 1901; Phdr., 243b3; Cra., 399a5; Euthph., 9a2, ed. 1900; Grg., 487d1; Men., 93e3, 1903; R., 502d4; Ti., 21c1.

${ }^{36}$ Cf. Pl., Ap., Tht. y $R$.
} 
bien puede ser conceptualizada como бoфía en un sistema filosófico que la avale como una cualidad que sirve al propósito de la verdad y del bien, como en el ejemplo de Antístenes. Éste no es el caso en un sistema filosófico, como el de Platón, donde la versatilidad y la astucia son competencias deseables, pero no suponen un compromiso con la verdad y el bien.

De esta manera determinamos cómo dichos atributos se excluyen de la conceptualización de ooфía, aclarando que la falta de tratamiento sistemá-

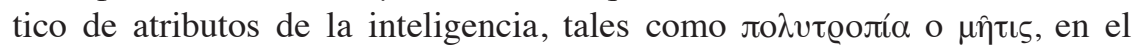
corpus platónico no indica forzosamente censura o condena en nombre de la moral o la verdad. En la medida en que estos atributos se presentan como cualidades mentales, no es que carezcan de valor, sino que únicamente tienen valor instrumental. Al comparar el caso de Antístenes con el de Platón, observamos que, si bien ambos filósofos vinculan la ooфía con la verdad y el bien, la filosofía platónica no lo hace desde las condiciones o características del sujeto que conoce, con sus dotes naturales, sino desde la naturaleza (divina y eterna) del objeto que, dentro de su filosofía, se aspira a conocer.

\section{BIBLIOGRAFÍA}

\section{Fuentes antiguas}

Aristophanes, Clouds, ed. Kenneth J. Dover, Oxford, Clarendon Press, 1968.

ARISTOTELIS Ethica Nicomachea, recognovit brevique adnotatione critica instruxit Ingram Bywater, Oxford, Clarendon Press, 1894.

Aristotelis Metaphysica, 2 vols., ed. William David Ross, Oxford, Clarendon Press, 1924.

Aristotelis Opera, vol. 12, Select fragments, ed. William David Ross, Oxford, Clarendon Press, 1952.

Die Fragmente der Vorsokratiker, vol. 1, ed. Hermann Diels and Walther Kranz, Berlin, Weidmann, 1951 (6th edn.).

Diogenis LaertiI Vitae philosophorum, ed. Herbert Strainge Long, Oxford, Clarendon Press, 1966.

EuRIPIDIS Fabulae, vol. 3, ed. Gilbert Murray, Oxford, Clarendon Press, 1902.

Herodoti Historiae, vol. 1, ed. Philippe-Ernest Legrand, Paris, Les Belles Lettres, 1932.

HipPocratis De Vetere Medicina, vol. 2, ed. Jacques Jouanna, Paris, Les Belles Lettres, 1990.

Homeri Odyssea, ed. Peter von der Mühll, Basel, Helbing \& Lichtenhahn, 1962.

Homeri Ilias, vol. 1, ed. Martin L. West, Stuttgart-Leipzig, Teubner (Bibliotheca Scriptorum Graecorum et Romanorum Teubneriana), 1998.

PINDARI Nemean Odes. Isthmian Odes. Fragments, ed. William H. Race, Cambridge, Harvard University Press, 1997. 
Pindari Olympian Odes. Pythian Odes, ed. William H. Race, Cambridge, Harvard University Press, 1997.

Platón, Diálogos, vol. IV, La República, intr., trad. y notas Conrado Eggers Lan, Madrid, Gredos, 1986.

Platonis Opera, vol. 1, Euthyphro, Apologia, Crito, Phaedo, Cratylus, Theaetetus, Sophista, Politicus, recognovit brevique adnotatione critica instruxit John Burnet, Oxford, Clarendon Press, 1900.

Platonis Opera, vol. 2, Parmenides, Philebus, Symposium, Phaedrus, Alcibiades 1 and 2, Hipparchus, Amatores, recognovit brevique adnotatione critica instruxit John Burnet, Oxford, Clarendon Press, 1901.

Platonis Opera, vol. 4, Clitopho, Respublica, Timaeus, Critias, recognovit brevique adnotatione critica instruxit John Burnet, Oxford, Clarendon Press, 1902.

Platonis Opera. vol. 3, Theages, Charmides, Laches, Lysis, Euthydemus, Protagoras, Gorgias, Meno, Hippias Maior, Hippias Minor, Io, Menexenus, recognovit brevique adnotatione critica instruxit John Burnet, Oxford, Clarendon Press, 1903.

Platonis Opera, vol. 5, Leges, recognovit brevique adnotatione critica instruxit John Burnet, Oxford, Clarendon Press, 1907.

Platonis Rempublicam, recognovit brevique adnotatione critica instruxit Simon Roelof Slings, Oxford, Oxford University Press, 2003.

PorPHYRII Quaestionum Homericarum ad Odysseam pertinentium reliquiae, textes, transl. et comm. Hermann Schrader, Leipzig, Teubner, 1890.

Senecae Epistulae, vol. 2, Epistles 66-92, transl. Richard M. Gummere, Cambridge, Harvard University Press, 1920.

Sophoclis Opera, vol. 2, ed. Alphonse Dain and Paul Mazon, Paris, Les Belles Lettres, 1958.

TheOGNIS Elegy and iambus: Being the remains of all the Greek elegiac and iambic poets from Callinus to Crates, excepting the choliambic writers, with the Anacreontes, ed. John Maxwell Edmonds, London, Heinemann, 1931.

\section{Fuentes modernas}

ADAms, Don, “Socrates polutropos?”, Apeiron, 43/1, 2010, pp. 33-61.

Barnouw, Jeffrey, Odysseus, Hero of Practical Intelligence. Deliberation and Signs in Homer's Odyssey, Lanham, University Press of America, 2004.

Blondell, Ruby, The Play of Character in Plato's Dialogues, Cambridge, Cambridge University Press, 2002.

Blundell, Mary Whitlock, "Character and Meaning in Plato's Hippias Minor", in James Carl Klagge y Nicholas D. Smith (eds.), Methods of Interpreting Plato and His Dialogues, Oxford, Clarendon Press, 1992, pp. 131-172.

Brancacci, Aldo, Oikeios logos. La filosofia del linguaggio di Antistene, Napoli, Bibliopolis, 1990.

Burnyeat, Myles, "Episteme", in Benjamin Morison and Katerina Ierodiakonou (eds.), Episteme, etc.: Essays in honour of Jonathan Barnes, Oxford, Oxford University Press, 2011, pp. 3-29. 
Detienne, Marcel, and Jean-Pierre Vernant, Cunning Intelligence in Greek Culture and Society, transl. Janet Lloyd, Hassocks, Harvester Press, 1978.

Dover, Kenneth James, Greek Popular Morality in the Time of Plato and Aristotle, Oxford, Blackwell, 1974.

FORD, Andrew L., The Origins of Criticism: Literary Culture and Poetic Theory in Classical Greece, Princeton, Princeton University Press, 2002.

García Gual, Carlos, Los siete sabios (y tres más), Madrid, Alianza Editorial, 2018. IrwIn, Terence, "Socrates and the Tragic Hero", in Pietro Pucci (ed.), Language and the Tragic Hero: Essays in Greek Tragedy in Honor of Gordon M. Kirkwood, Atlanta, Scholars Press, 1988, pp. 55-83.

Kofman, Sara, "Beyond Aporia", in Andrew Benjamin (ed.), Post-Structuralist Classics, London, Rutledge, 1988, pp. 7-44.

Lloyd, Geoffrey Ernest Richard, The Revolutions of Wisdom: Studies in the Claims and Practice of Ancient Greek Science, Berkeley, University of California Press, 1987.

Mársico, Claudia, Filósofos socráticos. Testimonios y fragmentos II. Antístenes, Fedón, Esquines y Simón, Buenos Aires, Editorial Losada, 2011.

Montiglio, Silvia, From Villain to Hero: Odysseus in Ancient Thought, Ann Arbor, University of Michigan Press, 2011.

Nehamas, Alexander, "Eristic, Antilogic, Sophistic, Dialectic: Plato's Demarcation of Philosophy from Sophistry", History of Philosophy Quarterly, 7/1, 1990, pp. 3-16.

Nightingale, Andrea Wilson, Genres in Dialogue: Plato and the Construct of Philosophy, Cambridge, Cambridge University Press, 1995.

Nightingale, Andrea Wilson, "Sages, Sophists, and Philosophers: Greek Wisdom Literature", in Oliver Taplin (ed.), Literature in the Greek and Roman Worlds: A New Perspective, Oxford, Oxford University Press, 2000, pp. 156-191.

RYLE, Gilbert, "Knowing how and knowing that: the presidential address", Proceedings of the Aristotelian Society, 46/1, 1945, pp. 1-16.

RYLE, Gilbert, The Concept of Mind, Harmondsworth, Penguin, 1949.

Silva, Trinidad, Naming the Wise: the Sophos, the Philosophos and the Sophistes in Plato, thesis for the degree of PhD in Classics, London, University College London, 2017.

Snowdon, Paul F., "Rylean Arguments: Ancient and Modern", in John Bengson and Marc A. Moffett (eds.), Knowing how: Essays on Knowledge, Mind and Action, Oxford, Oxford University Press, 2011, pp. 59-79.

Stanley, Jason, Know How, Oxford, Oxford University Press, 2011.

Tell, Hakan, Plato's Counterfeit Sophists, Washington, Center for Hellenic Studies (Hellenic Studies, 44), 2011.

Todd, Stephen, The Shape of Athenian Law, Oxford, Clarendon Press, 1993.

Wallace, Robert W., "Private Lives and Public Enemies: Freedom of Thought in Classical Athens", in Alan Boegehold and Adele Scafuro (eds.), Athenian Identity and Civic Ideology, Baltimore-London, The Johns Hopkins University Press, 1994, pp. 205-238.

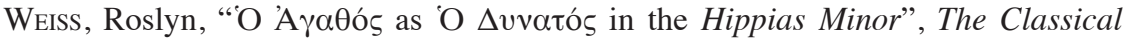
Quarterly, 31/2, 1981, pp. 287-304. 
Trinidad Silva Irarrázaval obtuvo el título de licenciada en Filosofía en la Pontificia Universidad Católica de Chile (2008), el de Máster en Estudios Clásicos (2011) y el de PhD en Filosofía Antigua (2017) en el Departamento de Greek \& Latin del University College London. Fue profesora asociada en el Departamento de Filosofía, de la Facultad de Filosofía de la Universidad de Barcelona (2017-2018) y actualmente es profesora e investigadora en la Facultad de Filosofía de la Universidad Alberto Hurtado, Chile. Sus líneas de investigación son particularmente Platón, los filósofos socráticos y escuelas helenísticas. 